\title{
Dinuclear system model in spontaneous fission process
}

\author{
Ivan Rogov ${ }^{1,2, *}$, Gurgen Adamian ${ }^{2}$, and Nikolai Antonenko ${ }^{2}$ \\ ${ }^{1}$ Tomsk Polytechnic University, 634050 Tomsk, Russia \\ ${ }^{2}$ Joint Institute for Nuclear Research, 141980 Dubna, Russia
}

\begin{abstract}
The application of the dinuclear system model is discussed to describe the spontaneous fission process. Cluster decay widths and spontaneous fission of ${ }^{234} \mathrm{U}$ are calculated within the same approach. The model describes well the experimental data on half-lives.
\end{abstract}

\section{Introduction}

After the emission of ${ }^{14} \mathrm{C}$ by ${ }^{223} \mathrm{Ra}$ was discovered [1, 2], other cluster radioactivities have been observed. Nowadays, $\alpha$-decay, cluster radioactivity (CR), and spontaneous fission (SF) attract attention in connection with the studies of the structure of heavy and superheavy nuclei. One of the most challenging problems is to describe the SF and $\mathrm{CR}$ processes in a single approach with the same parameters.

The model presented here belongs to the cluster type [3-10]. As assumed, the ground state of nucleus has a small admixture of the cluster-state components [11-16]. In Refs. [9, 10], a good description of the CR has been achieved. The natural step is to check if our model is able to describe the SF.

\section{Model}

In the model, the cluster configuration is represented as a dinuclear system (DNS), which can be described with the distance $R$ between the centers of mass of the clusters and charge asymmetry coordinate

$$
\eta_{Z}=\frac{Z_{H}-Z_{L}}{Z_{H}+Z_{L}}
$$

Here, $Z_{i}\left(A_{i}\right)$ is the charge (mass) number of the $i$-th cluster ( $i=L, H$ for light and heavy cluster, respectively). The mass asymmetry coordinate

$$
\eta=\frac{A_{H}-A_{L}}{A_{H}+A_{L}}
$$

is assumed to be strongly related to $\eta_{Z}$ by the condition of the potential energy minimum. Motion in $\eta_{Z}$ corresponds to cluster configuration formation; motion in $R$ coordinate describes the decay process.

Therefore, the decay width in the certain channel is proportional to the product of the probability $S_{L}$ of the DNS formation and the probability of decay of this DNS.

\footnotetext{
*e-mail: isrogov@theor.jinr.ru
}

The determination of $S_{L}$ requires the solution of the stationary Schrödinger equation:

$$
H \Psi_{n}\left(\eta_{z}\right)=E_{n} \Psi_{n}\left(\eta_{z}\right),
$$

where the collective Hamiltonian

$$
H=-\frac{\hbar^{2}}{2} \frac{\partial}{\partial \eta_{z}}\left(B^{-1}\right)_{\eta_{z}} \frac{\partial}{\partial \eta_{z}}+U\left(R, \eta_{z}\right),
$$

contains the inertia coefficient $\left(B^{-1}\right)_{\eta_{z}}$ [17] and the potential energy $U\left(R, \eta_{z}\right)$ [18]. The potential of the DNS [18]

$$
U\left(R, \eta_{Z}, \Omega\right)=V\left(R, \eta_{Z}, \Omega\right)-\left(B-B_{L}-B_{H}\right),
$$

is referred to a driving potential. Here, $B$ is the mass excess of the mother nucleus and $B_{L}, B_{H}$ are the mass excesses of the nuclei forming the DNS considered. The potential energy (5) is normalized with respect to the potential energy of mother-nucleus.

The nucleus-nucleus interaction potential $V$ is represented as the sum:

$$
V\left(R, \eta_{Z}, \Omega\right)=V_{\mathrm{C}}\left(R, \eta_{Z}\right)+V_{N}\left(R, \eta_{Z}\right)+V_{r}\left(R, \eta_{Z}, \Omega\right),
$$

of the Coulomb $V_{\mathrm{C}}$, nuclear $V_{N}$, and centrifugal $V_{r}$ potentials. Here, $\Omega$ is the orbital angular momentum. The form of $V$ for the ${ }^{28} \mathrm{Mg}+{ }^{206} \mathrm{Hg}$ system is presented in Fig. 1 .

To solve Eq. (3) and find $S_{L}$, it is convenient to replace $\eta_{Z}$ by

$$
x=2 \frac{Z_{L}}{Z}=1-\eta_{Z}
$$

This replacement of variables preserves the form of Eq. (3) with the function $\Psi(x)$ domain change to $x \in(0,1)$, where $x=0$ corresponds to the state of mononucleus, and $x=1$ notes the symmetric DNS configuration.

Using the replacement (7), one can extend the values of $U$ and $\left(B^{-1}\right)_{\eta_{Z}}$ to the segments of the width $2 \Delta=2 / Z$ so that the points $x_{U, B}$ are placed in the middle of the corresponding segments. The only exception is the mononucleus, for which we set $x \in[0,4 \Delta)$ and the $\alpha$-particle with $x \in[4 \Delta, 5 \Delta]$. 


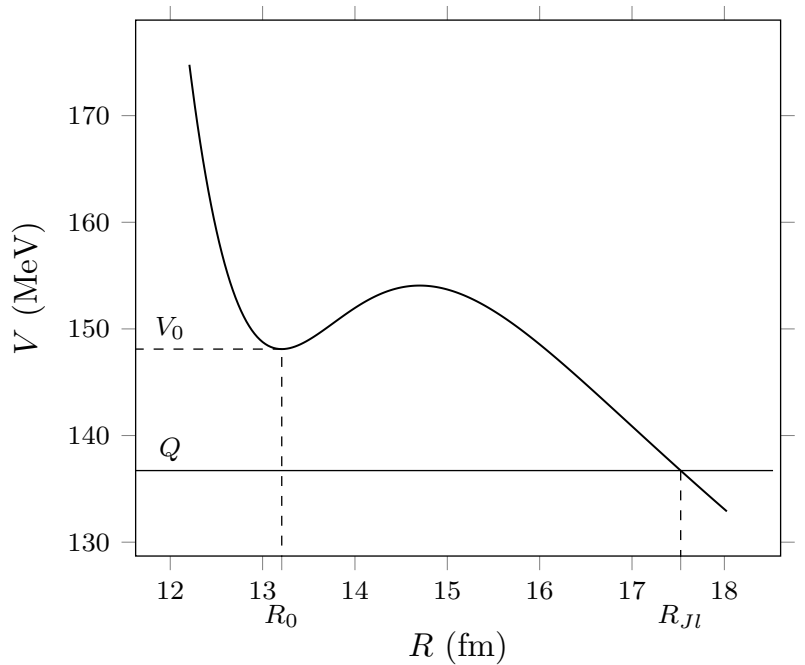

Figure 1. Calculated nucleus-nucleus potential for the ${ }^{28} \mathrm{Mg}+{ }^{206} \mathrm{Hg}$ system. The bottom of the potential pocket corresponds to $V_{0}$ at $R=R_{0}$. The position of external turning point corresponding to $V=Q$ is shown as $R_{J L}$.

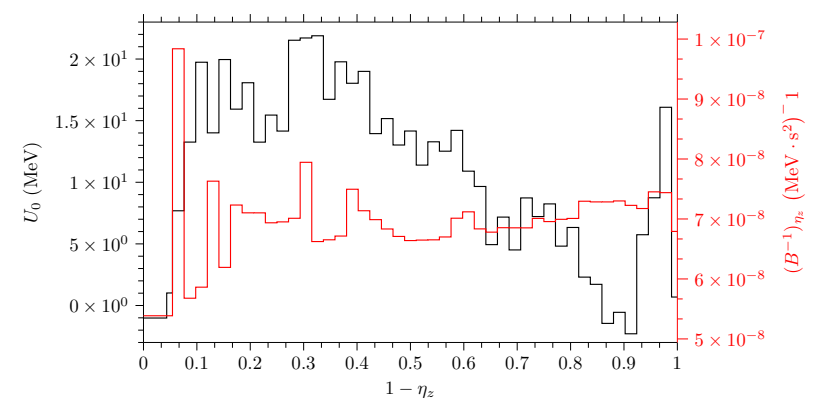

Figure 2. Calculated driving potential $U$ and inverse mass parameter $\left(B^{-1}\right)_{\eta_{Z}}$ as the step functions of $x$ for ${ }^{234} U$.

As seen in Fig. 2, there are local minima of the driving potentials at $\eta_{Z}$ close to zero. The potential energies in these minima are smaller than the energy of mononucleus at $\eta_{Z}=1$. Thus, the energy resolved configurations appear for the SF. These DNS configurations are excited.

The Schrödinger equation with the stepwise potential can be directly solved [19]. Using the solution of Eq. (3), we define the preformation probability $S_{L}$ of the DNS with certain charge number $Z_{L}$ of light cluster as follows:

$$
S_{L}=\int_{\eta_{Z}\left(Z_{L}\right)-\Delta}^{\eta_{Z}\left(Z_{Z}\right)+\Delta}\left|\Psi\left(\eta_{Z}\right)\right|^{2} d \eta_{Z}
$$

The value of the spectroscopic factor $S_{L}$ can not be directly measured. Only the experimental half-lives $T_{1 / 2}$ with respect to the $\mathrm{CR}$ and SF are available to be compared with the theoretical estimates. Thus, to compare the theoretical $T_{1 / 2}$ for the $\mathrm{CR}$, the probability $P_{L}$ of penetration through the Coulomb barrier is calculated in the one-
Table 1. Calculated and experimental alpha, cluster and spontaneous fission half-lives for ${ }^{234} \mathrm{U}$

\begin{tabular}{llll}
\hline & $\alpha$ & $\mathrm{CR}$ & $\mathrm{SF}$ \\
\hline$T_{1 / 2}^{\text {theor }}, \mathrm{s}$ & $5.02 \times 10^{12}$ & $3.00 \times 10^{25}$ & $9.11 \times 10^{22}$ \\
$T_{1 / 2}^{\text {exp }}, \mathrm{s}$ & $7.74 \times 10^{12}$ & $1.20 \times 10^{25}$ & $4.73 \times 10^{23}$ \\
\hline
\end{tabular}

dimensional WKB approximation

$$
P_{L}=\left(1+\exp \left[\frac{2}{\hbar} \int_{R_{0}}^{R_{J l}} \sqrt{2 \mu\left(V\left(R, \eta_{Z}\right)-Q\right)} d R\right]\right)^{-1}
$$

where $R_{0}$ and $R_{J l}$ are marked in Fig. $1, Q$ is the decay energy. The value of $T_{1 / 2}$ depends on the products $S_{L} P_{L}$. So, the decay width $\Gamma_{L}$ is calculated as

$$
\Gamma_{L}=\frac{\hbar \omega_{0}}{\pi} S_{L} P_{L}
$$

where $\omega_{0}$ is the frequency of zero-point vibration in $\eta_{z}$ coordinate near the mononucleus state, $\hbar \omega_{0}$ is equal to the distance between the ground and the first excited state of DNS. Using the formulas presented, the half-lives are calculated as

$$
T_{1 / 2}=\frac{\hbar \ln 2}{\Gamma_{L}}=\frac{\pi \ln 2}{\omega_{0} S_{L} P_{L}}
$$

\section{Calculated results}

To verify the model, the half-lives with respect to $\alpha$-decay, CR, and SF for ${ }^{234} \mathrm{U}$ were calculated (Table 1) and compared with the experimental data [20,21]. A good agreement between the theory and experiment has been obtained.

\section{Summary}

The model was developed to describe simultaneously the $\mathrm{CR}$ and SF. The CR, including $\alpha$-decay was considered as the evolution of the system in the collective coordinates of charge (mass) asymmetry and in the relative distance between the centers of clusters. The SF is considered in the same manner. The decays of almost symmetric DNS configurations are attributed to the SF. Indeed, the SF mainly occurs from the DNS configurations with $\eta_{Z}$ close to zero and corresponding to the minima of the driving potential. Calculating the penetrability of the barrier in the nucleusnucleus potential, the probability of DNS decay in $R$ is taken into account in the CR decay width.

The model presented describes well the half-lives of different decay channels for ${ }^{234} \mathrm{U}$.

\section{References}

[1] H.J. Rose and G.A. Jones, Nature 307, 245 (1984)

[2] D.V. Alexandrov et al., JETP Lett. 40, 909 (1984)

[3] Yu.M. Tchuvil'sky, Cluster Radioactivity (Moscow State University, Moscow, 1997) 
[4] W. Greiner, M. Ivascu, D.N. Poenaru, and A. Sandulescu Treatise on Heavy Ion Science (Plenum, New York, 1989)

[5] D.N. Poenaru et al., At. Data Nucl. Data Tables 34, 423 (1986); ibid 48, 231 (1991)

[6] Yu.S. Zamyatnin et al., Sov. J. Part. Nucl. 21, 231 (1990)

[7] S.G. Kadmensky, S.D. Kurgalin, Yu.M. Tchuvil'sky, Phys. Part. Nucl. 38, 699 (2007)

[8] D.N. Poenaru, Nuclear Decay Modes (IOP Publ., Bristol, 1996)

[9] S.N. Kuklin, G.G. Adamian, and N.V. Antonenko, Phys. At. Nucl. 68, 1443 (2005)

[10] S.N. Kuklin, G.G. Adamian, and N.V. Antonenko, Phys. Rev. C 71, 014301 (2005)

[11] T.M. Shneidman, G.G. Adamian, N.V. Antonenko, R.V. Jolos, and W. Scheid, Phys. Lett. B 526, 322 (2002)

[12] G.G. Adamian, N.V. Antonenko, R.V. Jolos, and T.M. Shneidman, Phys. Rev. C 70, 064318 (2004)
[13] G.G. Adamian, N.V. Antonenko, R.V. Jolos, Yu.V. Palchikov, and W. Scheid, Phys. Rev. C 67, 054303 (2003)

[14] G.G. Adamian, N.V. Antonenko, R.V. Jolos, Yu.V. Palchikov, W. Scheid and T.M. Shneidman, Phys. Rev. C 69, 054310 (2004)

[15] T.M. Shneidman, G.G. Adamian, N.V. Antonenko, and R.V. Jolos, Phys. Rev. C 74, 034316 (2006)

[16] G.G. Adamian, N.V. Antonenko, and W. Scheid, Lect. Notes Phys. 848, 165, (2012)

[17] G.G. Adamian, N.V. Antonenko, and R.V. Jolos, Nucl. Phys. A 584, 205 (1995)

[18] G.G. Adamian et al., Int. J. Mod. Phys. E 5, 191 (1996)

[19] C. Jirauschek, IEEE J. Quantum Electron. 45, 1059 (2009)

[20] J.K. Tuli, Nuclear wallet cards (NNDC, Brookhaven National Laboratory, 2011)

[21] H.R. von Gunten, A. Grütter, H.W. Reist, M. Baggenstos, Phys. Rev. C 23, 1110 (1981) 